\title{
Dynamics of periodic mechanical structures containing bistable elastic elements: From elastic to solitary wave propagation
}

\author{
Neel Nadkarni, ${ }^{1}$ Chiara Daraio,,${ }^{1,2}$ and Dennis M. Kochmann ${ }^{1, *}$ \\ ${ }^{1}$ Graduate Aerospace Laboratories, California Institute of Technology, Pasadena, California 91125, USA \\ ${ }^{2}$ Department of Mechanical and Process Engineering, ETH Zurich, Zurich, Switzerland
}

(Received 5 January 2014; published 22 August 2014)

\begin{abstract}
We investigate the nonlinear dynamics of a periodic chain of bistable elements consisting of masses connected by elastic springs whose constraint arrangement gives rise to a large-deformation snap-through instability. We show that the resulting negative-stiffness effect produces three different regimes of (linear and nonlinear) wave propagation in the periodic medium, depending on the wave amplitude. At small amplitudes, linear elastic waves experience dispersion that is controllable by the geometry and by the level of precompression. At moderate to large amplitudes, solitary waves arise in the weakly and strongly nonlinear regime. For each case, we present closed-form analytical solutions and we confirm our theoretical findings by specific numerical examples. The precompression reveals a class of wave propagation for a partially positive and negative potential. The presented results highlight opportunities in the design of mechanical metamaterials based on negative-stiffness elements, which go beyond current concepts primarily based on linear elastic wave propagation. Our findings shed light on the rich effective dynamics achievable by nonlinear small-scale instabilities in solids and structures.
\end{abstract}

DOI: 10.1103/PhysRevE.90.023204

PACS number(s): 42.65.Tg, 46.32.+x, 46.40.-f, 62.20.D-

\section{INTRODUCTION}

The periodic arrangement of small-scale building blocks results in acoustic or mechanical metamaterials [1], which have attracted great attention because of their extremely rich field of applications including acoustic wave guides and filters [2,3], acoustic lenses and diodes [4,5], sound isolators and sensors [6,7], and acoustic cloaks and sonar stealth technologies $[8,9]$. Design strategies commonly exploit the scattering of elastic waves in periodic media at characteristic frequencies in all or specific directions [10-12] as well as resonant phenomena capable of absorbing energy on lower scales by local resonators $[5,13,14]$. In all these examples, the careful microscale periodic architecture of multiscale engineered material systems leads to an interesting or beneficial effective dynamic behavior on the macroscale. Besides pronounced acoustic band gaps $[15,16]$, this design paradigm has resulted in negative effective dynamic stiffness [17] and mass density [18,19] and combinations of both [20]. Here negative stiffness and negative mass density refer to the effective dynamic properties: An elastic system containing only positive-stiffness elements can demonstrate negative effective dynamic quantities near resonance. All these phenomena in the linear elastic regime are well understood.

Static negative (incremental) stiffness arises from instabilities in solids and structures when the energy landscape loses (some notion of) convexity. Bistable elements such as the spring configuration schematically shown in Fig. 1 make an excellent example: The potential energy's nonconvexity provides the system with an unstable regime of negative energy curvature (i.e., negative incremental stiffness) whose nonlinear force-displacement relation leads to a spontaneous snapping from one stable equilibrium to the next energy minimum if pure tractions are applied. The same phenomenon can be observed in prestressed buckled structural members [21].

\footnotetext{
*kochmann@ caltech.edu
}

Furthermore, solids undergoing phase transitions or domain switching display a similar instability on the microscale when the crystal lattice transforms from one energy-minimizing phase into another (or into a mixture of variants) under the action of external stimuli such as thermal [22,23] or electric fields [24,25] or under imposed deformation or applied loads $[26,27]$. In all systems, a negative curvature of the energy landscape implies a violation of positive definiteness of the incremental elastic modulus tensor, which has been referred to as (static) negative stiffness [28,29].

While unconstrained homogeneous solids with static negative stiffness are thermodynamically unstable [30], the possibility to stabilize negative stiffness in heterogeneous media (e.g., non-positive-definite phases in linear elastic composite materials [31,32]) has become a focus of scientific interest due to the exciting effective physical properties that may arise from the combination of positive- and negative-stiffness phases in a composite system. Examples include extreme values of the effective viscoelastic moduli $[29,33]$, the piezo- and pyroelectric as well as thermoelastic coefficients [34], and extreme effective dynamic moduli [35]. The effective quasistatic and dynamic behavior of systems containing negative-stiffness phases has been subject to substantial investigation both theoretically [31,36-38] and experimentally [23,29,39-41]. However, such studies to date have focused on the linear elastic regime.

The rich dynamics of physical, chemical, and biological systems containing bistable elements have been studied extensively, demonstrating, among other things, the formation of solitary waves. Previous studies on the nonlinear dynamics of discrete systems have used various phenomenological potentials of the Frenkel-Kontorova form giving rise to a kink soliton solution. For example, periodic potentials of Sine-Gordon type, nonsinusoidal and multibarrier potentials [42-45], and anharmonic potentials have been used to model such diverse physical systems as dislocation dynamics [46-50], incommensurate structural transitions in surface physics [51-53] and dielectrics [54,55], ferromagnetic and 


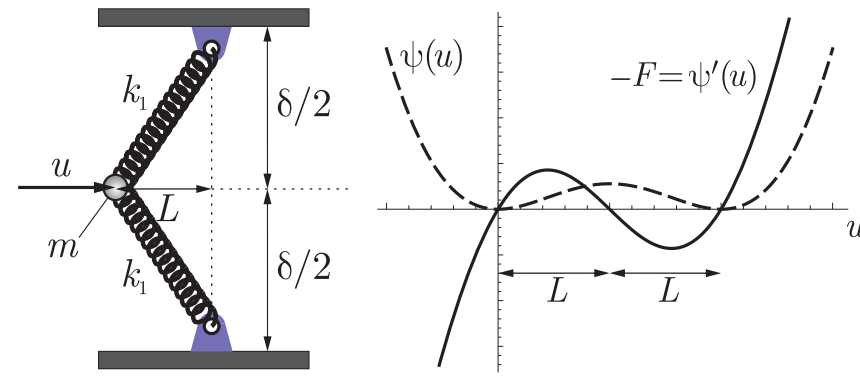

FIG. 1. (Color online) Bistable element consisting of two elastic springs and a point mass, energy $\psi(u)$, and force $F(u)$.

antiferromagnetic domain wall transitions [56-58], and molecular shock interactions $[59,60]$, to mention but a few. The mechanical response of carbon nanotubes [61,62] as well as of cellular structural solids [63], has been explained on the basis of buckling or snapping instabilities arising from systems with multiple stable configurations. Moreover, the dynamics of atomic-scale configurational changes as well as the mechanisms of damage and fracture have been analyzed by recourse to chains of elements with a nonmonotonic force-displacement (or stress-strain) response; see, e.g., Refs. [64-71].

The dynamic response of such nonmonotonic force chains was shown theoretically to result in solitary waves $[44,45,72-$ 74]. In addition, previous results have shown that chains of elements with nonmonotonic (piecewise-linear) forcedisplacement relations can dissipate energy at a fast rate by transforming kinetic energy into high-frequency oscillations (so-called twinkling modes). This was later extended to chains with smooth nonmonotonic force-displacement relations in a comprehensive stability analysis [75]. It was also experimentally demonstrated in chains of granular particles with a nonsmooth contact interaction [76]. Other relevant research has focused on the quasistatic response, including the derivation of atomically informed stress-strain relations [77] or studies of the hysteresis and dissipative effects in elastoplasticity [78,79].

Although the beneficial effects of negative-stiffness elements on the effective dynamic performance of acoustic or mechanical metamaterials and composites is well known [38], previous research has focused on the linear elastic regime. However, as discussed above, bistable or multistable building blocks in periodic solids and structures also promise interesting nonlinear dynamic effects, including solitary-wave propagation, which provides opportunities to focus acoustic signals in mechanical metamaterials $[4,80,81]$. Homogeneous solids undergoing finite elastic deformation [82] as well as periodic media experiencing nonlinear elastic instabilities [83] have been shown to exhibit acoustic band gaps that are controllable by the amount of nonlinear predeformation, yet the investigated waves again operate in the linear elastic regime. To date, only one example of a periodic elastic mechanical system has been reported that produces SineGordon solitons by allowing a kink propagation in the form of elastically connected rotating pendulums [84-88]. The weakly or strongly nonlinear response of elastic media containing negative-stiffness elements such as the bistable spring configuration shown in Fig. 1 has remained widely unexplored, in part because such instabilities in solids and the resulting nonlinear effective dynamics are mathematically complex and make analytical solutions a rare find.

Here we study a mechanical system capable of propagating impact pressure waves in three different regimes, serving as a model for the creation of nonlinear acoustic metamaterials with static negative-stiffness elements. We present closed-form analytical results for the nonlinear response of a chain of bistable elements consisting of elastic springs and point masses. The specific configuration of the periodically repeated elementary unit cell displays a continuous nonmonotonic force-displacement relation with two stable equilibria and one unstable equilibrium configuration giving rise to temporary negative (static) stiffness. We have deliberately chosen a simple albeit instructive mechanical system that enables us to study the rich dynamics of periodic chains of bistable elements in the full range of its linear to strongly nonlinear behavior. The chosen spring configuration shows the same features as prebuckled structures (while allowing for a clean analytical investigation) and the conclusions drawn here can qualitatively be transferred to numerous structural instabilities. In fact, the interesting wave propagation characteristics reported here hint at the design of novel mechanical metamaterials with controllable wave propagation in the linear and nonlinear regimes, with applications ranging from waveguides and amplifiers to vibration attenuators. The chosen system admits a clean identification of all model parameters and allows for experimental implementation. We note that in our analysis we assume conservative systems and thereby neglect energy dissipation through internal friction or other damping mechanisms that are usually found in mechanical systems. Of course, the presence of damping will alter the response of the system (our numerical examples contain small amounts of damping to remove transient effects, whereas all analytical solutions assume energy conservation).

\section{BISTABLE CHAIN CONFIGURATION}

\section{A. Geometry and kinetics}

Consider the bistable structure consisting of two identical and symmetrically arranged linear elastic springs with stiffness $k_{1}$ as shown schematically in Fig. 1 in its unstressed equilibrium configuration. Both springs are connected by a joint allowing for rotation and carrying point mass $m$. Their free ends are attached to joints, which allow for rotation but prevent translation. Due to symmetry, a horizontal force on mass $m$ will result in a horizontal displacement $u$, so we may treat the system as one dimensional in the following. The total potential energy stored by the structure is given by

$$
\psi(u)=k_{1}\left[l(u)-l_{0}\right]^{2},
$$

where

$$
l(u)=\sqrt{(L-u)^{2}+(\delta / 2)^{2}}
$$

is the deformed length of each spring with geometric details $\delta$ and $L$ introduced in Fig. 1. Consequently, the initial spring length is given by $l_{0}=l(0)$. Energy (1) is illustrated in Fig. 1 as a function of the displacement $u$, which demonstrates two stable equilibria (i.e., local energy minima) and one unstable equilibrium configuration (corresponding to the local energy 


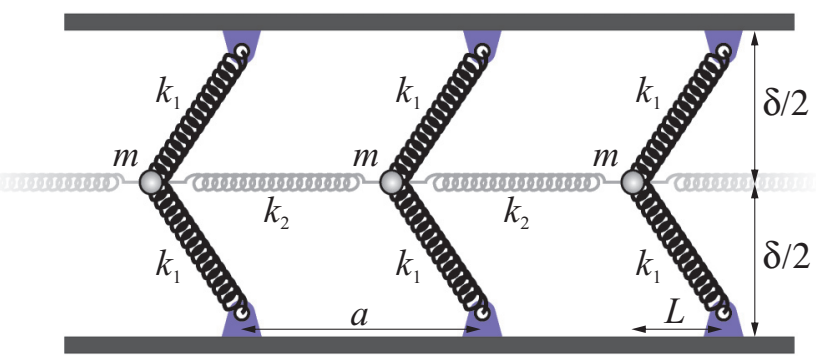

FIG. 2. (Color online) Periodic chain of bistable elements.

maximum). The structure experiences negative stiffness as it passes through this unstable regime.

To arrive at a periodic one-dimensional chain, we consider an array of $N$ such bistable elements connected by horizontal linear springs of stiffness $k_{2}$ that act as force transfer elements. Identical masses $m$ are placed at each node of the chain, as shown schematically in Fig. 2. The total Hamiltonian of the spring system can be written as

$$
H\left(\boldsymbol{u}, \boldsymbol{u}_{, t}\right)=\sum_{i=1}^{N}\left[\frac{m}{2} u_{i, t}^{2}+\psi_{i}\left(u_{i}\right)\right]+\sum_{i=1}^{N-1} \frac{k_{2}}{2}\left(u_{i+1}-u_{i}\right)^{2},
$$

where $\psi\left(u_{i}\right)$ is the potential energy stored by the $i$ th bistable pair of springs, $\boldsymbol{u}=\left\{u_{1}, \ldots, u_{N}\right\}$ denotes the vector of all nodal displacements depending on time $t$, and a comma in indices denotes differentiation with respect to the ensuing variable(s). Therefore, the rate of change of momentum of mass $i$ follows from Hamilton's equation, i.e.

$$
p_{i, t}=m u_{i, t t}=-\frac{\partial H}{\partial u_{i}} .
$$

Application of (3) yields

$$
m u_{i, t t}+k_{2}\left(-u_{i+1}+2 u_{i}-u_{i-1}\right)-F\left(u_{i}\right)=0,
$$

where the internal force applied to mass $i$ by the bistable spring element was introduced as

$$
F(u)=-\psi^{\prime}(u)=-2 k_{1}(L-u) \frac{l(u)-l_{0}}{l(u)} .
$$

\section{B. Scaling and continuum limit}

Dimensionless equations will enable us to investigate the mechanics of the system without having to specify geometry and material parameters explicitly. In order to reduce the problem to a self-similar type, we scale the displacements and the nodal spacing with respect to their respective length scales. For a lattice parameter $a$ (initial spacing between two masses), we define

$$
\bar{x}=\frac{x}{a}, \quad \bar{u}=\frac{u}{L},
$$

where $\bar{x}$ and $\bar{u}$ are the dimensionless $x$ coordinate and displacement, respectively. Therefore, the force $F(u)$ can be expressed in dimensionless form as

$$
\bar{F}(\bar{u})=\frac{F(u)}{k_{1} L}=2(1-\bar{u})\left(1-\frac{\bar{l}_{0}}{\bar{l}(u)}\right)
$$

with

$$
\bar{l}(\bar{u})=\sqrt{(1-\bar{u})^{2}+d^{2}}, \quad d=\frac{\delta}{2 L}, \quad \bar{l}_{0}=\bar{l}(0) .
$$

This suggests that the dimensionless force of the bistable spring element only depends on ratio $d=\delta / 2 L$ and not on actual lengths. The governing equation (5) can be nondimensionalized by using the same force scale and defining two new dimensionless parameters, viz., the stiffness ratio $K_{r}$ and the characteristic time scale $T$ (the period associated with the eigenfrequency of mass $m$ attached to a single spring of stiffness $k_{1}$ ):

$$
K_{r}=\frac{k_{2}}{k_{1}}, \quad T=\sqrt{\frac{m}{k_{1}}} .
$$

This gives

$$
\bar{u}_{i, \bar{t}}+K_{r}\left(-\bar{u}_{i+1}+2 \bar{u}_{i}-\bar{u}_{i-1}\right)-\bar{F}\left(\bar{u}_{i}\right)=0 .
$$

We choose the origin such that mass $i$ is initially located at $x_{i}=i a$ and positions are normalized by defining

$$
\bar{x}_{i}=\frac{x_{i}}{a}=i \quad \text { so that } \quad \Delta \bar{x}=\bar{x}_{i}-\bar{x}_{i-1}=1 .
$$

In the continuum limit $(a \rightarrow 0), \bar{u}_{i+1}$ and $\bar{u}_{i-1}$ can be written, using a Taylor expansion, as

$$
\bar{u}_{i \pm 1}=\bar{u}_{i} \pm \frac{\partial \overline{u_{i}}}{\partial \bar{x}}+\frac{1}{2} \frac{\partial^{2} \overline{u_{i}}}{\partial \bar{x}^{2}} \pm \frac{1}{6} \frac{\partial^{3} \overline{u_{i}}}{\partial \bar{x}^{3}}+\frac{1}{24} \frac{\partial^{4} \overline{u_{i}}}{\partial \bar{x}^{4}}+\mathcal{T},
$$

where $\mathcal{T}$ denotes higher-order terms. By substituting (13) into (11), we obtain the dimensionless continuum limit of the equation of motion

$$
\bar{u}_{, \bar{t}}-K_{r}\left(\frac{\partial^{2} \bar{u}}{\partial \bar{x}^{2}}+\frac{1}{12} \frac{\partial^{4} \bar{u}}{\partial \bar{x}^{4}}+\mathcal{T}\right)-\bar{F}(\bar{u})=0 .
$$

\section{Dispersion relation and long-wavelength approximation}

In order to determine the dispersion relation of the periodic chain in the continuum limit, let us first consider the linear regime. Here the equation of motion can be approximated by linearizing (8) about $\bar{u}=0$, which gives

$$
\bar{u}_{, \bar{t} \bar{t}}-K_{r}\left(\frac{\partial^{2} \bar{u}}{\partial \bar{x}^{2}}+\frac{1}{12} \frac{\partial^{4} \bar{u}}{\partial \bar{x}^{4}}+\mathcal{T}\right)+\omega_{0}^{2} \bar{u}=0,
$$

where

$$
\omega_{0}^{2}=\frac{2}{1+d^{2}} .
$$

We assume a traveling-wave solution of the form

$$
\bar{u}=\hat{u} e^{i(\bar{q} \bar{x}-\bar{\omega} \bar{t})},
$$

with $\bar{q}=q a$ and $\bar{\omega}=\omega T$. Substitution of (17) into (15) yields the continuum dispersion relation

$$
\bar{\omega}(\bar{q})=\sqrt{\omega_{0}^{2}+K_{r}\left(\bar{q}^{2}-\frac{1}{12} \bar{q}^{4}+\mathcal{T}\right)} .
$$

In the long-wavelength limit $\bar{q}=q a \ll 1$ the dispersion relation can be approximated by only retaining the leadingorder quadratic term and dropping all higher-order terms such that (18) becomes

$$
\bar{\omega}(\bar{q}) \approx \bar{\omega}_{c}(\bar{q})=\sqrt{\omega_{0}^{2}+K_{r} \bar{q}^{2}} .
$$




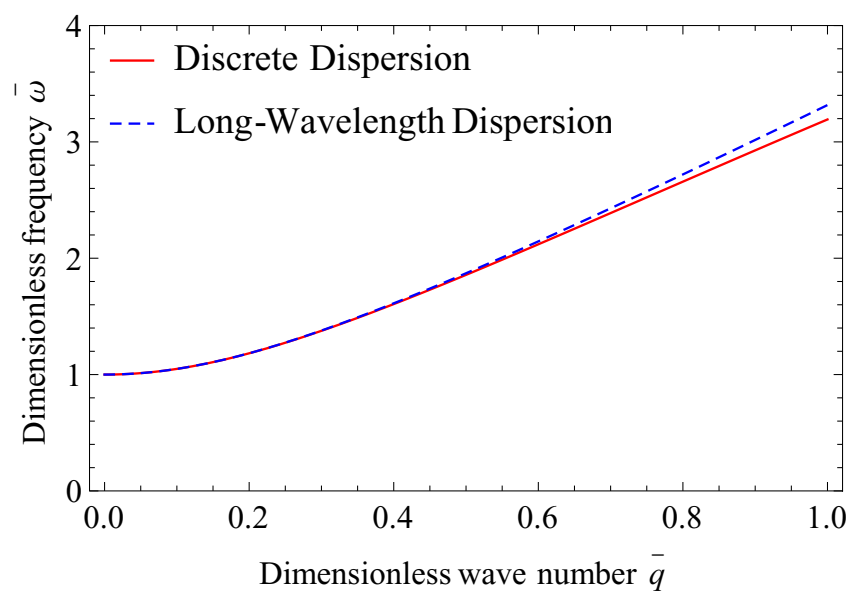

FIG. 3. (Color online) Dispersion relation comparison.

The exact dispersion relation for the discrete system is obtained by substituting the traveling wave form (17) into the discrete equation of motion (11), which gives

$$
\bar{\omega}_{d}(\bar{q})=\sqrt{\omega_{0}^{2}+2 K_{r}(1-\cos \bar{q})} .
$$

Obviously, in the long-wavelength limit $\bar{q} \ll 1$ [keeping only quadratic terms in (20)], the two dispersion relations (19) and (20) agree. Figure 3 compares the exact discrete and the approximate continuum dispersion relations (for $K_{r}=10$ ) and demonstrates excellent agreement with deviations of less than $3 \%$ up to $\bar{q}=0.86$. Therefore, in the following we only consider long wavelengths, for which the governing equation assumes wave equation character (with dimensionless wave speed $\bar{c}_{0}^{2}=K_{r}$ ):

$$
\bar{u}_{, \bar{t} \bar{t}}-\bar{c}_{0}^{2} \bar{u}_{\bar{x} \bar{x}}-\bar{F}(\bar{u})=0 .
$$

\section{Regimes of wave propagation}

Owing to the nonlinearity of force $\bar{F}(\bar{u})$, we can identify three distinct regimes of wave propagation that depend on the magnitude of the amplitude of $\bar{u}$ and for each case we seek solutions for the propagating wave by approximating the nonlinear force in (21). We discriminate the following three regimes of propagating waves: (i) For small amplitudes $\bar{u} \ll 1$ we expand the nonlinear spring force to linear leading order as

$$
\bar{F}(\bar{u}) \approx-\frac{2}{1+d^{2}} \bar{u}=-\omega_{0}^{2} \bar{u},
$$

(ii) for moderate amplitudes $\bar{u}<1$ we approximate the nonlinear force by a third-order Taylor expansion, i.e.,

$$
\bar{F}(\bar{u}) \approx-\frac{2}{1+d^{2}} \bar{u}+\frac{3 d^{2}}{\left(1+d^{2}\right)^{2}} \bar{u}^{2}-\frac{d^{2}\left(d^{2}-4\right)}{\left(1+d^{2}\right)^{3}} \bar{u}^{3},
$$

and (iii) for large amplitudes $\bar{u}>1$ we use the exact spring force

$$
\bar{F}(\bar{u})=2(1-\bar{u})\left(1-\frac{\bar{l}_{0}}{\bar{l}(u)}\right) .
$$

Figure 4 illustrates the three approximations of the nonlinear spring force in comparison with the exact force for the specific choice of $d=1$. We note that the nodal spacing $a$

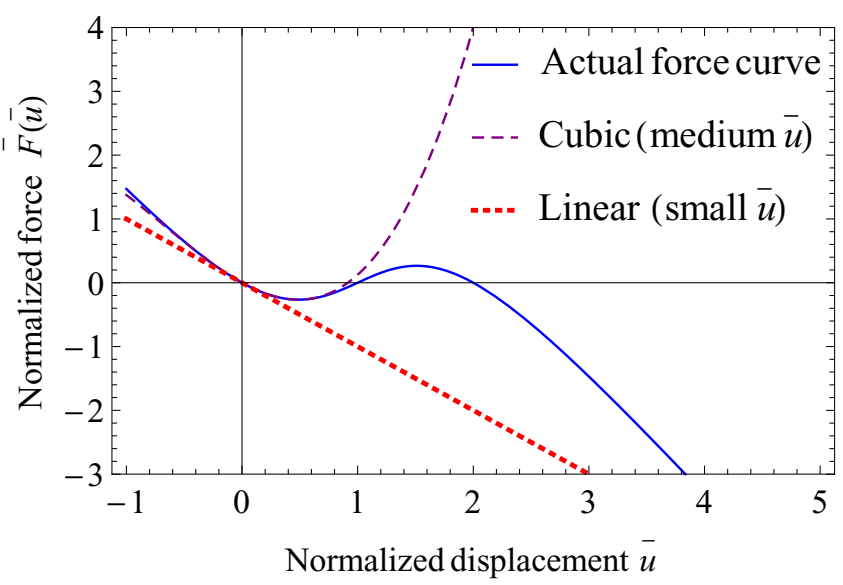

FIG. 4. (Color online) Nonlinear spring force and approximations introduced for the three regimes for $d=1$.

is independent of the characteristic spring length $L$ and of ratio $d$, so in the long-wavelength limit the governing equation for all three regimes is given by (21). The special case of amplitude $\bar{u}=1$ is excluded because $\bar{u}=1$ corresponds to an unstable equilibrium configuration of the system. The smallest perturbation is sufficient to cause the system to snap into either energy well and thus to transform the scenario into either the $\bar{u}>1$ or the $\bar{u}<1$ case.

In the following sections we will investigate the wave propagation behavior in all three regimes. To confirm our theoretical solutions, we will compare to numerical results obtained for the example parameters $d=1, T=1$, and $K_{r}=$ 10 , from which the three regimes are chosen as (i) $|\bar{u}| \leqslant 0.05$, (ii) $|\bar{u}| \leqslant 0.3$, and (iii) $|\bar{u}| \leqslant 2$. Convincing agreement has been verified for various combinations of these parameters; for brevity we present here only this specific case.

Numerical solutions are obtained from a chain of 100 elementary unit cells modeled in the time domain by an implicit finite-difference scheme of Newmark- $\beta$ type with parameters chosen to minimize numerical damping $(\beta=$ 0.25 and $\gamma=0.5$ ). Displacement and/or velocity boundary conditions are directly imposed on the first node of the chain, while the remaining nodes may vibrate freely. All nodes are constrained to only move horizontally.

\section{WAVE PROPAGATION RESULTS}

\section{A. Small amplitude: Linear solution}

\section{Analytical solution}

The equation governing the wave propagation in this regime is given by

$$
\bar{u}_{, \bar{t} \bar{t}}-\bar{c}_{0}^{2} \bar{u}_{\bar{x} \bar{x}}+\omega_{0}^{2} \bar{u}=0,
$$

with $\omega_{0}^{2}$ from (16). This is the dimensionless linear KleinGordon equation [89] for the unknown displacement field $\bar{u}(\bar{x}, \bar{t})$. The theoretical solution for this problem is of the form

$$
\bar{u}(\bar{x}, \bar{t})=A \cos (\bar{q} \bar{x}-\bar{\omega} \bar{t})+B \sin (\bar{q} \bar{x}-\bar{\omega} \bar{t}),
$$

where the dimensionless wave number $\bar{q}$ and the dimensionless angular frequency $\bar{\omega}$ are related by the dispersion relation (19). Therefore, this regime admits the propagation of linear elastic 


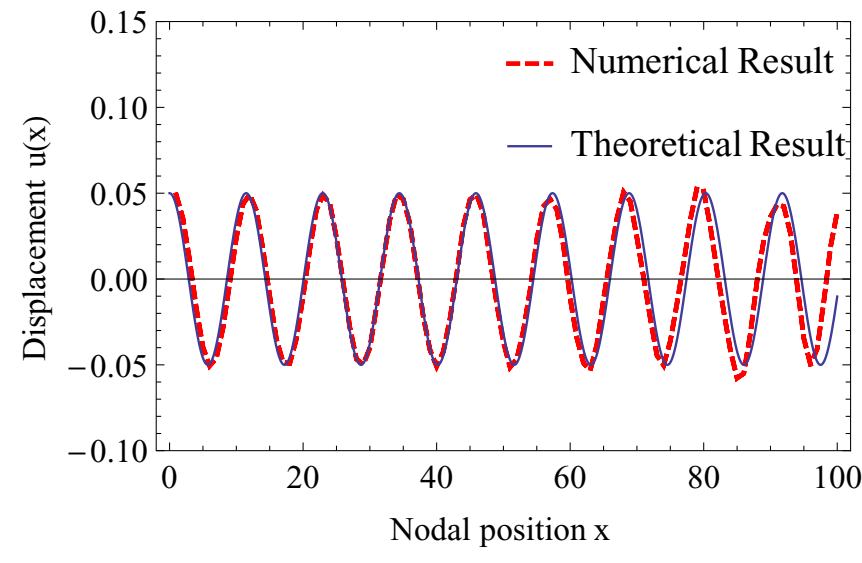

FIG. 5. (Color online) Small-amplitude regime: numerical results compared to the linear Klein-Gordon solution.

waves at frequencies outside the stop bands characterized by the dispersion relations.

\section{Numerical results}

For the numerical benchmark test, the first node of the chain of bistable elements is excited by time-harmonic displacements (we enforce displacement and corresponding velocity boundary conditions at the first node) according to

$$
\bar{u}_{1}(t)=\hat{u} \cos (\bar{\omega} \bar{t}), \quad \bar{u}_{1, t}(t)=-\hat{u} \bar{\omega} \sin (\bar{\omega} \bar{t}) .
$$

As only small amplitudes are permitted in this regime, we choose $\hat{u}=0.05$ along with a value of $\bar{\omega}=2$ such that the long-wavelength limit holds, which becomes apparent from the dispersion relations in Fig. 3. Transient effects are removed by artificial initial damping.

Figure 5 shows a comparison of the numerical solution with the theoretical solution in space, at a specific instant of time. The two solutions match with minor deviations due to numerical noise arising from the discrete solution (as the wave passes through the long chain towards its rightmost node, a weakly nonlinear effect causes the curve to slightly deviate from the theoretical solution). The wave speed can be inferred from the $x$ - $t$ contour diagram of the numerical solution shown in Fig. 6. The observed velocity of propagation agrees with the phase speed computed from the dispersion relation (also included as a solid red line). Nonlinear effects therefore only play a negligible role in this regime of small amplitudes, so the linear Klein-Gordon solution is a legitimate approximation to describe and explain the wave propagation behavior in this regime.

\section{B. Medium amplitude: Weak nonlinearity}

\section{Analytical solution}

For moderate displacements, the dimensionless governing equation in the continuum long-wavelength limit is approximated by

$$
\bar{u}_{\bar{t} \bar{t}}-\bar{c}_{0}^{2} \bar{u}_{\bar{x} \bar{x}}+\frac{2}{1+d^{2}} \bar{u}-\frac{3 d^{2}}{\left(1+d^{2}\right)^{2}} \bar{u}^{2}+\frac{d^{2}\left(d^{2}-4\right)}{\left(1+d^{2}\right)^{3}} \bar{u}^{3}=0,
$$

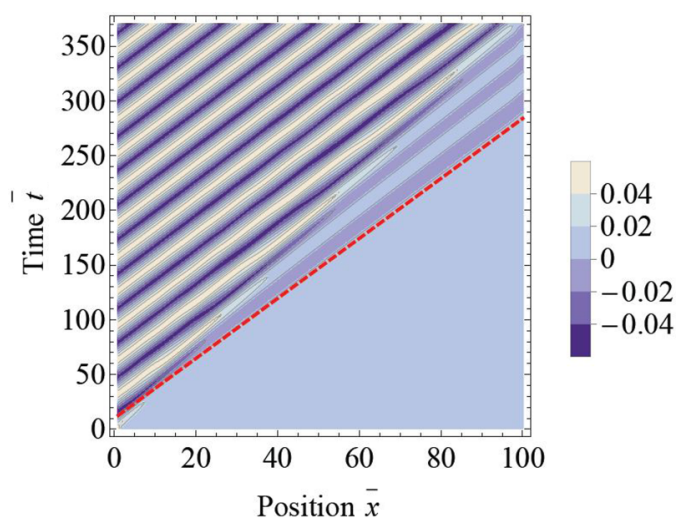

FIG. 6. (Color online) Small-amplitude regime: $x$ - $t$ contour diagram of the numerical solution; for comparison, the solid red line represents a positive characteristic of the theoretical solution.

an equation of cubic nonlinear Klein-Gordon type [90,91] for the unknown displacement field $\bar{u}(\bar{x}, \bar{t})$. The solution can be found by a perturbation multiple-scale expansion [92]. Therefore, we use the ansatz

$$
\bar{u}(\bar{x}, \bar{t})=\epsilon \phi_{0}(\bar{x}, \bar{t})+\epsilon^{2} \phi_{1}(\bar{x}, \bar{t})+\epsilon^{3} \phi_{2}(\bar{x}, \bar{t})+O\left(\epsilon^{4}\right),
$$

where $|\epsilon| \ll 1$ is a small characteristic length scale. For the current problem, the expansion is restricted to third order, since this approximation demonstrates sufficient accuracy for the medium amplitude regime; cf. Sec. II D. Suppose that in addition to variables $\bar{x}$ and $\bar{t}$ the solution depends on multiple scales of position and time. Then new scaled variables can be defined by

$$
X_{i}=\epsilon^{i} \bar{x}, \quad T_{i}=\epsilon^{i} \bar{t} .
$$

Again, we limit scales to order 3. Consequently, we now seek solutions

$$
\phi_{i}(\bar{x}, \bar{t})=\phi_{i}\left(X_{0}, X_{1}, X_{2}, T_{0}, T_{1}, T_{2}\right) .
$$

Derivatives with respect to the primary variables become

$$
\frac{\partial}{\partial \bar{t}}=\sum_{i=0}^{2} \epsilon^{i} D_{i}, \quad \frac{\partial}{\partial \bar{x}}=\sum_{i=0}^{2} \epsilon^{i} D_{X i},
$$

where we introduced operators

$$
D_{i}=\frac{\partial}{\partial T_{i}}, \quad D_{X i}=\frac{\partial}{\partial X_{i}} .
$$

Substitution of the chosen representation of the displacement field (27) into the governing equation (23) and inspecting the equation at $O(\epsilon)$ leads to

$$
\mathcal{L} \phi_{0}=0 \quad \text { with } \mathcal{L}=D_{0}^{2}-\bar{c}_{0}^{2} D_{X 0}^{2}+\omega_{0}^{2} .
$$

Hence, the solution for the first-order expansion is of the form

$$
\phi_{0}=A\left(X_{1}, X_{2}, T_{1}, T_{2}\right) e^{i\left(\bar{q} X_{0}-\bar{\omega} T_{0}\right)}+\text { c.c. }
$$

Here and in the following c.c. stands for the complex conjugate (for conciseness we do not write out both terms; the complex conjugate terms follow analogously). Frequency $\bar{\omega}$ and wave number $\bar{q}$ are related by the first-order dispersion relation for 
small amplitudes discussed above. At $O\left(\epsilon^{2}\right)$, the governing equation reads

$$
\begin{gathered}
\left(D_{0}+\epsilon D_{1}\right)^{2}\left(\epsilon \phi_{0}+\epsilon^{2} \phi_{1}\right)-\bar{c}_{0}^{2}\left(D_{X 0}+\epsilon D_{X 1}\right)^{2}\left(\epsilon \phi_{0}+\epsilon^{2} \phi_{1}\right) \\
+\frac{2}{1+d^{2}}\left(\epsilon \phi_{0}+\epsilon^{2} \phi_{1}\right)-\frac{3 d^{2}}{\left(1+d^{2}\right)^{2}}\left(\epsilon \phi_{0}+\epsilon^{2} \phi_{1}\right)^{2}=0,
\end{gathered}
$$

which can be algebraically reduced to the simpler form

$$
\mathcal{L} \phi_{1}=-\left(2 D_{0} D_{1}-2 c_{0}^{2} D_{X 0} D_{X 1}\right) \phi_{0}+\frac{3 d^{2}}{\left(1+d^{2}\right)^{2}} \phi_{0}^{2} .
$$

Substitution of (33) into (34) and defining $\sigma=\bar{q} X_{0}-\bar{\omega} T_{0}$ results in

$$
\begin{aligned}
\mathcal{L} \phi_{1}= & \left(2 i \bar{\omega} \frac{\partial A}{\partial T_{1}}+2 i \bar{q}{\overline{c_{0}}}^{2} \frac{\partial A}{\partial X_{1}}\right) e^{i \sigma} \\
& +\frac{3 d^{2}}{\left(1+d^{2}\right)^{2}} A^{2} e^{i 2 \sigma}+\frac{3 d^{2}}{\left(1+d^{2}\right)^{2}}|A|^{2}+\text { c.c. }
\end{aligned}
$$

For simplicity the overbars on $c_{0}^{2}, q$, and $\omega$ will be dropped henceforth. Because $\sigma$ is the eigenfrequency of the operator $\mathcal{L}$, the coefficient of the resonant term cancels, implying

$$
\omega \frac{\partial A}{\partial T_{1}}+q c_{0}^{2} \frac{\partial A}{\partial X_{1}}=0
$$

By introducing the group velocity $v_{g}=q c_{0}^{2} / \omega$ (which can be verified from the dispersion relation), we know that function $A$ must be of the form

$$
A\left(X_{1}, X_{2}, T_{1}, T_{2}\right)=A\left(X_{1}-v_{g} T_{1}, X_{2}, T_{2}\right) .
$$

Therefore, (35) reduces to

$$
\mathcal{L} \phi_{1}=\frac{3 d^{2}}{\left(1+d^{2}\right)^{2}} A^{2} e^{i 2 \sigma}+\frac{3 d^{2}}{\left(1+d^{2}\right)^{2}}|A|^{2}+\text { c.c. }
$$

The inhomogeneous solution is determined from the ansatz

$$
\begin{aligned}
\phi_{1}= & B\left(X_{1}, X_{2}, T_{1}, T_{2}\right) e^{2 i \sigma}+C\left(X_{1}, X_{2}, T_{1}, T_{2}\right) \\
& + \text { c.c. },
\end{aligned}
$$

which is substituted into (38). As $A$ does not depend on $X_{0}$ and $T_{0}$, the nonoscillating terms corresponding to the absolute value cancel on both sides. Hence, only the resonant terms remain:

$$
\begin{aligned}
& \left(-4 \omega^{2}+4{\overline{c_{0}}}^{2} q^{2}+\omega_{0}^{2}\right) B e^{2 i \sigma}+\omega_{0}^{2} C \\
& =\frac{3 d^{2}}{\left(1+d^{2}\right)^{2}} A^{2} e^{i 2 \sigma}+\frac{3 d^{2}}{\left(1+d^{2}\right)^{2}}|A|^{2}+\text { c.c. }
\end{aligned}
$$

Solving for $B$ and $C$ (ignoring complex conjugates) results in

$$
B=-\frac{d^{2}}{2\left(1+d^{2}\right)}, \quad C=\frac{3 d^{2}}{2\left(1+d^{2}\right)}|A|^{2},
$$

which implies that

$$
\phi_{1}=-\frac{d^{2}}{2\left(1+d^{2}\right)} A^{2} e^{i 2 \sigma}+\frac{3 d^{2}}{2\left(1+d^{2}\right)}|A|^{2}+\text { c.c. }
$$

Similarly at $O\left(\epsilon^{3}\right)$, we have

$$
\begin{aligned}
& \left(D_{0}+\epsilon D_{1}+\epsilon^{2} D_{2}\right)^{2}\left(\epsilon \phi_{0}+\epsilon^{2} \phi_{1}+\epsilon^{3} \phi_{2}\right) \\
& -c_{0}^{2}\left(D_{X 0}+\epsilon D_{X 1}+\epsilon^{2} D_{1}+\epsilon^{3} \phi_{2}\right)^{2}\left(\epsilon \phi_{0}+\epsilon^{2} \phi_{1}+\epsilon^{3} \phi_{2}\right)
\end{aligned}
$$

$$
\begin{aligned}
& +\omega_{0}^{2}\left(\epsilon \phi_{0}+\epsilon^{2} \phi_{1}+\epsilon^{3} \phi_{2}\right) \\
& +\frac{d^{2}\left(d^{2}-4\right)}{\left(1+d^{2}\right)^{3}}\left(\epsilon \phi_{0}+\epsilon^{2} \phi_{1}+\epsilon^{3} \phi_{2}\right)^{2} \\
& -\frac{6 d^{2}}{\left(1+d^{2}\right)^{2}}\left(\epsilon \phi_{0}+\epsilon^{2} \phi_{1}+\epsilon^{3} \phi_{2}\right)^{3}=0,
\end{aligned}
$$

which, after canceling appropriate terms, reduces to

$$
\begin{aligned}
\mathcal{L} \phi_{2}= & -\frac{d^{2}}{1+d^{2}}\left(2 A \frac{\partial A}{\partial T_{1}}(-i \omega)-c_{0}^{2} 2 A \frac{\partial A}{\partial X_{1}}(i q)\right) e^{i 2 \sigma} \\
& -\left(2 \frac{\partial A}{\partial T_{2}}(-i \omega)-2 c_{0}^{2} \frac{\partial A}{\partial X_{2}}(i q)+\frac{\partial^{2} A}{\partial T_{1}^{2}}-c_{0}^{2} \frac{\partial^{2} A}{\partial X_{1}^{2}}\right) e^{i \sigma} \\
& +\frac{12 d^{2}}{\left(1+d^{2}\right)^{2}}|A|^{2} A e^{i \sigma}-\frac{4 d^{2}\left(d^{2}-1\right)}{\left(1+d^{2}\right)^{3}} A^{3} e^{i 3 \sigma} .
\end{aligned}
$$

Due to (36), the coefficient of the $e^{i 2 \sigma}$ term vanishes. To ensure finite amplitudes, the resonant term proportional to $e^{i \sigma}$ must not contribute to the solution. Consequently, the only remaining term gives

$$
\mathcal{L} \phi_{2}=-\frac{4 d^{2}\left(d^{2}-1\right)}{\left(1+d^{2}\right)^{3}} A^{3} e^{i 3 \sigma}
$$

with the solution

$$
\phi_{2}=-\frac{d^{2}\left(d^{2}-1\right)}{4\left(1+d^{2}\right)^{2}} A^{3} e^{i 3 \sigma} .
$$

Since the coefficient of the resonant term in (44) must vanish, we also have

$$
\begin{gathered}
2 \frac{\partial A}{\partial T_{2}}(-i \omega)-2 c_{0}^{2} \frac{\partial A}{\partial X_{2}}(i q)+\frac{\partial^{2} A}{\partial T_{1}^{2}}-c_{0}^{2} \frac{\partial^{2} A}{\partial X_{1}^{2}} \\
-\frac{12 d^{2}}{\left(1+d^{2}\right)^{2}}|A|^{2} A=0 .
\end{gathered}
$$

Let us introduce the following new variables:

$$
\begin{array}{ll}
\xi_{1}=X_{1}-v_{g} T_{1}, & \eta_{1}=T_{1}, \\
\xi_{2}=X_{2}-v_{g} T_{2}, & \eta_{2}=T_{2} .
\end{array}
$$

Substitution into (47) and simplification finally yields (with overbars for the final solution)

$$
i \frac{\partial A}{\partial \eta_{2}}+\frac{\bar{c}_{0}^{2}-\bar{v}_{g}^{2}}{2 \bar{\omega}} \frac{\partial^{2} A}{\partial \xi_{1}^{2}}+\frac{6 d^{2}}{\bar{\omega}\left(1+d^{2}\right)^{2}}|A|^{2} A=0 .
$$

Equation (49) is a nonlinear Schrödinger (NLS) equation for the unknown function $A$. Therefore, $A$ scales with $\epsilon^{2}$ in time and with $\epsilon$ in space. In general, a NLS equation of the form

$$
i \frac{\partial A}{\partial \eta_{2}}+P \frac{\partial^{2} A}{\partial \xi_{1}^{2}}+Q|A|^{2} A=0
$$

with coefficients $P, Q \in \mathbb{R}$ has two types of solutions [89,93]:

$$
\begin{aligned}
& P Q>0 \Rightarrow \text { envelope solitons, } \\
& P Q<0 \Rightarrow \text { dark solitons. }
\end{aligned}
$$

In the current case, one can verify that

$$
P Q=\left(\frac{\bar{c}_{0}^{2}-\bar{v}_{g}^{2}}{2 \bar{\omega}}\right)\left(\frac{6 d^{2}}{\bar{\omega}\left(1+d^{2}\right)^{2}}\right)=\frac{3 c_{0}^{2} \omega_{0}^{2} d^{2}}{\bar{\omega}^{4}\left(1+d^{2}\right)^{2}}>0
$$


for all admissible values of $\bar{\omega}$. Therefore, the solution of the displacement field in this regime can be characterized as an envelope soliton [94], which is caused by a Benjamin-Feir modulational instability $[93,95]$ of the wave as it propagates through the system for $P Q>0$. A similar kind of modulational instability was observed in Sine-Gordon and $\phi^{4}$ problems [72]. The NLS equation can be solved exactly for function $A$ [96], giving

$$
A=V_{m} \operatorname{sech}\left(\sqrt{\frac{Q}{2 P}} \epsilon V_{m}\left(\bar{x}-v_{g} \bar{t}\right)\right) \exp \left(i\left(\epsilon V_{m}\right)^{2} \frac{Q}{2} \bar{t}\right),
$$

where $V_{m}$ is the characteristic amplitude of the wave and

$$
\frac{Q}{2 P}=\frac{6 d^{2} \bar{\omega}^{2}}{\bar{c}_{0}^{2} \omega_{0}^{2}\left(1+d^{2}\right)^{2}}, \quad \frac{Q}{2}=\frac{3 d^{2}}{\bar{\omega}\left(1+d^{2}\right)^{2}} .
$$

Therefore, at $O(\epsilon)$ the displacement field can be expressed as

$$
\bar{u}(\bar{x}, \bar{t})=\epsilon A(\bar{x}, \bar{t}) \exp (i \sigma)+\text { c.c. }
$$

\section{Numerical results}

We use the same numerical setup as before to simulate the wave propagation behavior in this regime, but we expand the number of nodes to 200 (to ensure sufficient level of detail to compare with the continuous solution). In order to see the formation of an envelope soliton due to modulational instability, a sinusoidal plane wave is imposed by the boundary conditions at the first node, viz., by enforcing

$$
\bar{u}_{1}(\bar{t})= \begin{cases}\hat{u} \cos (\bar{\omega} \bar{t}) & \text { for } 0 \leqslant \bar{t} \leqslant \bar{\tau} \\ 0 & \text { otherwise }\end{cases}
$$

and

$$
\bar{u}_{1, \bar{t}}(\bar{t})= \begin{cases}-\hat{u} \bar{\omega} \sin (\bar{\omega} \bar{t}) & \text { for } 0 \leqslant \bar{t} \leqslant \bar{\tau} \\ 0 & \text { otherwise. }\end{cases}
$$

Instead of fixing the displacement at the first node for $\bar{t} \notin[0, \bar{\tau}]$, one can leave the first node traction-free without significantly affecting the solution. The boundary node at the other end of the chain is kept traction-free for all times.

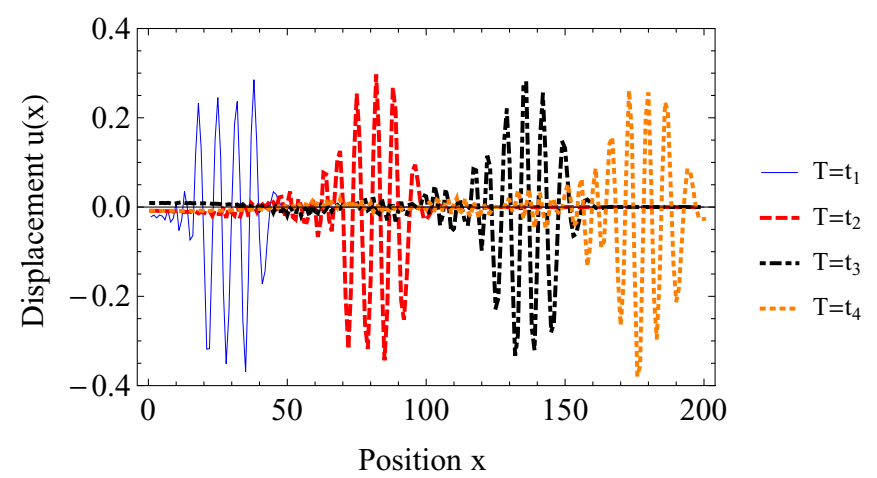

FIG. 7. (Color online) Snapshots of the propagating wave (traveling from left to right) at different instances of time show the evolution of the envelope soliton. The sech-type envelope begins to form due to self-modulation, as the wave passes through the lattice.

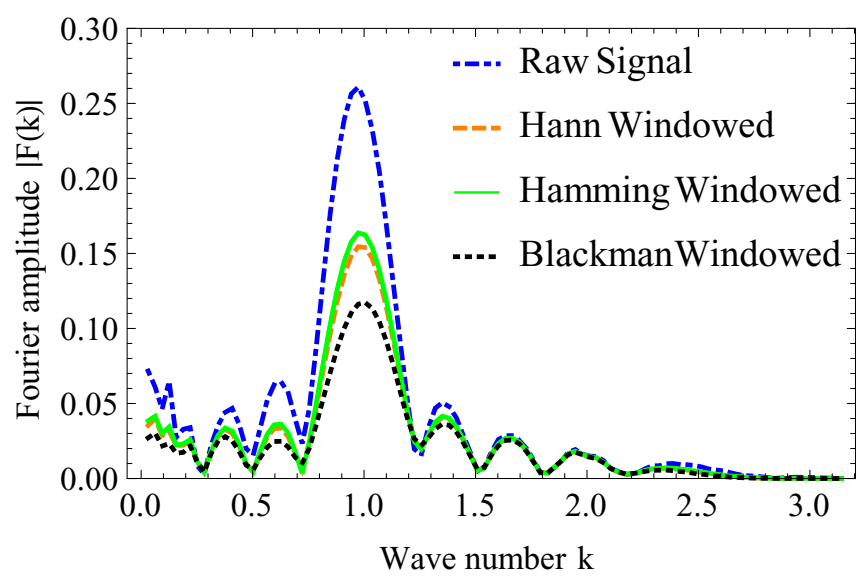

FIG. 8. (Color online) Discrete Fourier transform of the spatial variation of the waveform at a chosen instant of time.

Figure 7 illustrates numerical results for the specific choices of $\hat{u}=0.3, \bar{\omega}=3$, and $\bar{\tau}=10$. Results clearly confirm the propagation of a soliton, yet they show a variation of the envelope soliton as it moves through the lattice. The envelope of the waveform is seen to oscillate at a different frequency than the applied frequency at the first node. In order to verify the nature of the waveform, we show the Fourier spectrum in Fig. 8. In avoidance of spectral leakage due to the limitations of a discrete Fourier transform, Hann, Blackman, and Hamming windows are used to determine the spectral content of the signal. The resulting peak frequency corresponds to the spatial wave number of the applied frequency. However, side lobes form, which can be explained by the modulational instability [93] caused by the weakly nonlinear effects discussed above. Hence, wave propagation in this regime can indeed be explained by the nonlinear Schrödinger and the cubic nonlinear Klein-Gordon equations and numerical results confirm the theoretical prediction of an envelope soliton. The envelope appears to vary as it moves along the chain, yet the waveform preserves it localized nature, leaving behind small-amplitude high-frequency oscillations.

\section{Large amplitude: Strong nonlinearity}

\section{Analytical solution}

For the case of large amplitudes, we use the exact nonlinear form of the potential energy. Therefore, the governing equation in the continuum limit is

$$
\bar{u}_{\bar{t} \bar{t}}-\bar{c}_{0}^{2} \bar{u}_{\bar{x} \bar{x}}-\bar{F}(\bar{u})=0 .
$$

For convenience, the overbars are omitted in the following. We seek a traveling-wave solution of the form $u(x, t)=u(x-$ $v t)=u(z)$, where $v$ is the propagation velocity and $z=x-v t$ a reduced variable. Substitution into (58) gives

$$
\left(v^{2}-c_{0}^{2}\right) u_{z z}-F(u)=0 .
$$

Multiplication by $\frac{d u}{d z}$ and integration leads to

$$
\frac{v^{2}-c_{0}^{2}}{2}\left(\frac{d u}{d z}\right)^{2}+\psi(u)=C_{1},
$$


where $C_{1}$ is a constant of integration. The value of $C_{1}$ can be computed from the initial conditions

$$
\lim _{z \rightarrow \infty} u(z)=0, \quad \lim _{z \rightarrow \infty} \frac{d u(z)}{d z}=0,
$$

which translate into

$$
C_{1}=0
$$

Therefore, after some rearrangement (60) becomes

$$
\sqrt{\frac{c_{0}^{2}-v^{2}}{2}} \int \frac{d u}{\sqrt{\psi(u)}}=z-z_{0}
$$

where $z_{0}$ is another constant of integration. Performing the integration results in

$$
\begin{aligned}
& \frac{2}{\sqrt{1+d^{2}}} \ln \left(a(u)+\frac{a(u)}{b(u)} \sqrt{1+d^{2}}\right) \\
& +\ln \left(\frac{1-a(u)}{1+a(u)} \frac{1-b(u)}{1+b(u)}\right)=\sqrt{\frac{2}{c_{o}^{2}-v^{2}}}\left(z-z_{0}\right)
\end{aligned}
$$

with the abbreviations

$$
a(u)=u-1, \quad b(u)=\frac{\sqrt{1+d^{2}}(u-1)}{\sqrt{(u-1)^{2}+d^{2}}} .
$$

In summary, the solution in the large-amplitude case is indeed a propagating wave of type $u(x, t)=u(x-v t)$. From (64) we conclude that $c_{0}>v$, which implies that the wave speed is subsonic. Although (64) cannot be inverted to solve for $u(z)$ explicitly, the relation shows that $u(z) \rightarrow 0$ as $z \rightarrow \infty$ and that $u(z) \rightarrow 2$ as $z \rightarrow-\infty$. In addition, the function can be plotted parametrically for $u \in(0,2)$, which is shown in Fig. 9 . Obviously, the wave front is localized and of kink soliton type, which can be physically explained by the snap-through effect of each spring from one stable configuration to the other. The shape of the kink depends on the velocity of propagation with higher velocity kinks having steeper slopes.

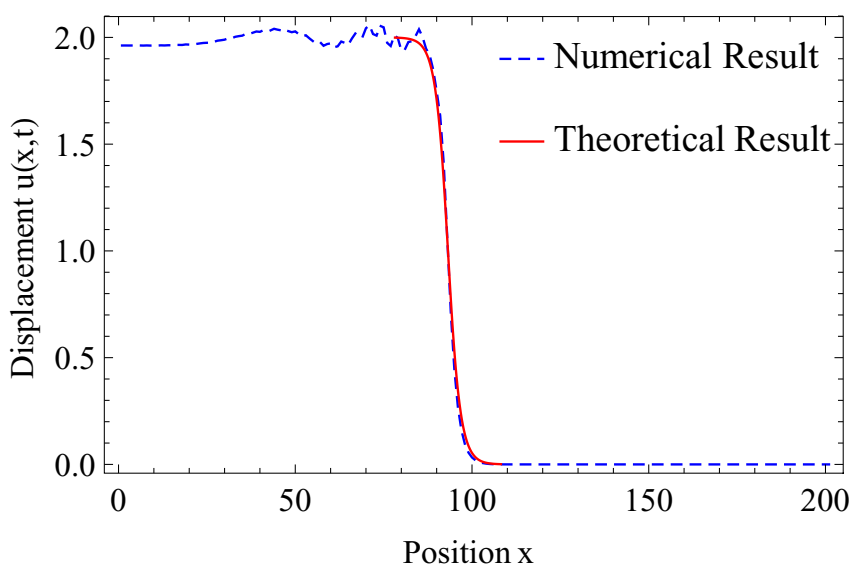

FIG. 9. (Color online) Comparison of the theoretical largeamplitude solution and numerically determined wave profile (for parameters $d=1, z_{0}=-93.2$, and $v=2.812$ ).

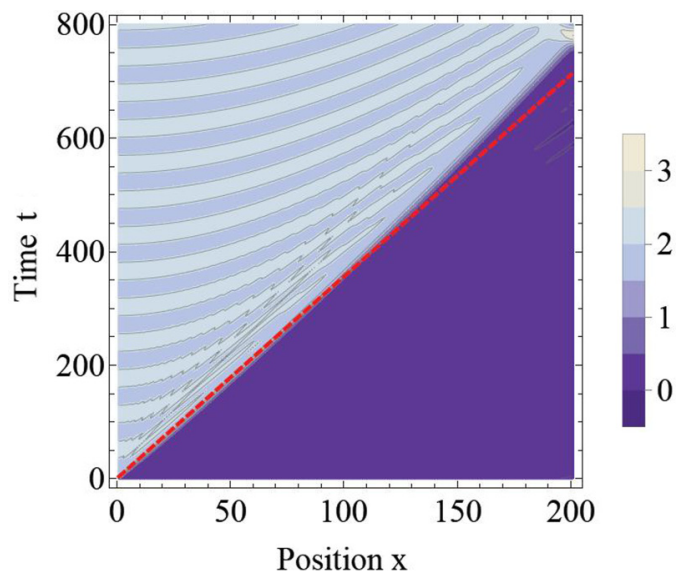

FIG. 10. (Color online) The $x$ - $t$ contour diagram of the numerical solution for the large-amplitude regime. The red straight line is the best-fit line corresponding to the leading edge characteristic.

\section{Numerical results}

We use the same numerical setup as before to simulate a chain of 100 bistable spring elements. We apply to the first node an initial velocity sufficiently high to make the first mass snap to its other stable branch. The other end of the chain is kept free at all times, i.e., zero tractions are enforced on that boundary. A comparison of the numerically determined wave profile and the theoretical exact solution propagating with the same velocity is shown in Fig. 9. The perturbations in the numerical solution with respect to the theoretical solution are caused due discreteness effects $[97,98]$. To allow for a direct comparison, the velocity of propagation in the numerical example is found by linearly fitting the position of the leading edge in the $x-t$ contour plot in Fig. 10. The linear fit is shown in Fig. 11, which shows that the velocity is not constant, but variations with time are marginal, so we may assume the wave is traveling at almost constant speed. Inserting the propagation velocity into the theoretical solution shows an excellent match with

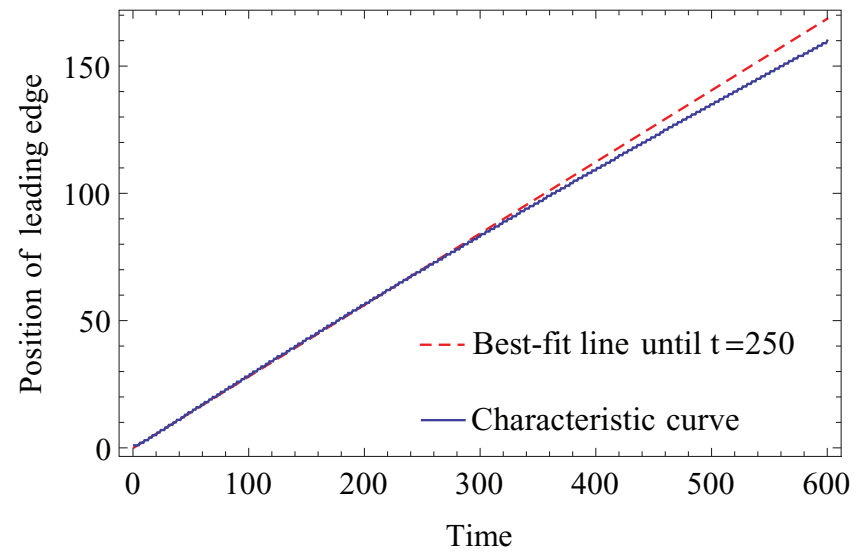

FIG. 11. (Color online) The characteristic curve corresponding to the leading edge of the wave is shown in comparison with the best-fit solution at $t=250$. The slope of the line determines the initial speed of the propagating wave. This speed is used to compute the exact solution in the kink soliton propagation. The kink slows down toward the end due to the energy radiated by the oscillatory tail. 
the numerical wave profile, which confirms the accuracy of the aforementioned analytical solution for the large-amplitude regime. The propagating wave is of antisoliton nature with a topological charge of -1 .

\section{Energy of the kink soliton}

Bistable elements have been shown to produce twinkling, which results in energy dissipation; see, e.g., Ref. [75] and references therein. Here we disregard just oscillations and focus on the propagating kink soliton whose energy can be determined by integrating the Hamiltonian spatial density over the complete lattice at any given time. The Hamiltonian density per unit spacing in the continuum limit is given by

$$
h(x, t)=u_{t}^{2}+\frac{1}{2} c_{0}^{2} u_{x}^{2}+\psi(u) .
$$

In the large-amplitude case as derived in Sec. III C 1, we have $u(x, t)=u(x-v t)=u(z)$. Substitution into (66) gives

$$
h(z)=\left(\frac{v^{2}+c_{0}^{2}}{2}\right) u_{z}^{2}+\psi(u) .
$$

Replacing the value of $\psi(u)$ by using (60), we arrive at

$$
h(z)=\left(\frac{v^{2}+c_{0}^{2}}{2}\right) u_{z}^{2}+\left(\frac{c_{0}^{2}-v^{2}}{2}\right) u_{z}^{2}=c_{0}^{2} u_{z}^{2} .
$$

Therefore, the total energy is computed by integrating $h(z)$ over the complete lattice, which results in

$$
\begin{aligned}
E & =\int_{-\infty}^{\infty} h(z) d z \\
& =\frac{c_{0}^{2}}{\sqrt{2\left(c_{0}^{2}-v^{2}\right)}}\left[d^{2} \ln \left(\frac{1+\sqrt{1+d^{2}}}{\sqrt{1+d^{2}}-1}\right)-2 \sqrt{1+d^{2}}\right] .
\end{aligned}
$$

Consequently, (69) implies that the energy increases with the propagation speed of the soliton. In combination with results from Sec. III C 1, we thus conclude that faster moving solitons have a steeper slope and have higher energy.

\section{Effect of precompression}

For the linear, weakly nonlinear, and strongly nonlinear regimes, we have shown how the wave characteristics (including the dispersion relations, wave speeds, and wave profiles) can be fine-tuned by changing the geometric parameter $d$. In addition, the nonlinearity of the bistable spring elements admits tailoring of the dynamic response of the chain by applying initial preloads. Specifically, assume identical constant forces $F_{0}$ are applied to all masses in their direction of motion (e.g., by arranging the chain of springs vertically and letting the point masses predeform the bistable spring elements under the action of gravity, while the connecting linear springs remain unstretched in equilibrium). Consequently, all masses exhibit an initial equilibrium displacement $u_{0}$ that satisfies

$$
F_{0}=-\psi^{\prime}\left(u_{0}\right) \text {. }
$$

Wave solutions are obtained by approximating the nonlinear force-displacement relation about this new initial equilibrium configuration. Let $u$ denote the displacement from the new equilibrium state so that the total force on a bistable spring element becomes

$$
F(u)=-\psi^{\prime}\left(u_{0}+u\right) .
$$

By using the dimensionless definitions of Sec. II B, we arrive at the equations of motion in the presence of preloads, viz.,

$$
\bar{u}_{i, \bar{t} \bar{t}}+K_{r}\left(-\bar{u}_{i+1}+2 \bar{u}_{i}-\bar{u}_{i-1}\right)-\bar{F}(\bar{u})+\bar{F}_{0}=0
$$

or, after substitution of (70) and (71),

$\bar{u}_{i, \bar{t} \bar{t}}+K_{r}\left(-\bar{u}_{i+1}+2 \bar{u}_{i}-\bar{u}_{i-1}\right)+\bar{\psi}^{\prime}\left(\bar{u}_{0}+\bar{u}_{i}\right)-\bar{\psi}^{\prime}\left(\bar{u}_{0}\right)=0$.

For small amplitudes $(\bar{u} \ll 1)$ we take

$$
\bar{\psi}^{\prime}\left(\bar{u}_{0}+\bar{u}_{i}\right) \simeq \bar{\psi}^{\prime}\left(\bar{u}_{0}\right)+\bar{\psi}^{\prime \prime}\left(\bar{u}_{0}\right) \bar{u}_{i},
$$

which again leads to a linearized Klein-Gordon equation, viz.,

$$
\bar{u}_{i, \bar{t} \bar{t}}+K_{r}\left(-\bar{u}_{i+1}+2 \bar{u}_{i}-\bar{u}_{i-1}\right)+\bar{\psi}^{\prime \prime}\left(\bar{u}_{0}\right) \bar{u}_{i}=0 .
$$

Therefore, the same solution derived above applies when defining

$$
\omega_{0}^{2}=\bar{\psi}^{\prime \prime}\left(\bar{u}_{0}\right)=2\left[1-\frac{d^{2} \sqrt{1+d^{2}}}{\left[d^{2}+\left(\bar{u}_{0}-1\right)^{2}\right]^{3 / 2}}\right],
$$

which naturally reduces to (16) when choosing $\bar{u}_{0}=0$. Therefore, predeformation $u_{0}$ can be utilized to manipulate the dispersion relation (19). We note that the amount of precompression is limited before snapping occurs. Specifically, (76) only yields real-valued wave speeds if

$$
u_{0} \leqslant 1-d \sqrt{\left(1+d^{-2}\right)^{1 / 3}} \text {. }
$$

Next let us consider moderate amplitudes. The applicability of this regime now depends on the predeformation. Here $\psi^{\prime}(\bar{u}+$ $\left.\overline{u_{0}}\right)-\psi^{\prime}\left(\overline{u_{0}}\right)=0$ generally has two solutions $\left\{\bar{u}^{*}, \bar{u}^{* *}\right\}$ with $\bar{u}^{*}<\bar{u}^{* *}$. In the following, we assume moderate amplitudes that satisfy $\bar{u}^{*}+\bar{u}_{0}<\bar{u}^{* *}$ to prevent snapping. A third-order Taylor expansion of the nonlinear spring force turns (73) into

$$
\begin{aligned}
& \bar{u}_{i, \bar{t} \bar{t}}+K_{r}\left(-\bar{u}_{i+1}+2 \bar{u}_{i}-\bar{u}_{i-1}\right)+\bar{\psi}^{\prime \prime}\left(\bar{u}_{0}\right) \bar{u}_{i} \\
& \quad+\frac{1}{2} \bar{\psi}^{\prime \prime \prime}\left(\bar{u}_{0}\right) \bar{u}_{i}^{2}+\frac{1}{6} \bar{\psi}^{\prime \prime \prime \prime}\left(\bar{u}_{0}\right) \bar{u}_{i}^{3}=0 .
\end{aligned}
$$

A multiple-scale expansion approach again leads to a nonlinear Schrödinger equation, which in turn gives rise to a wave profile of envelope soliton type as derived in Sec. III B, the details of which are omitted here due to the close analogy to the previous analysis.

Finally, in the case of large amplitudes we use the exact forcing function, which gives rise to the governing equation in the continuum limit of the following form:

$$
\bar{u}_{\bar{t} \bar{t}}-\bar{c}_{0}^{2} u_{\bar{x} \bar{x}}+\psi^{\prime}\left(\bar{u}+\bar{u}_{0}\right)-\psi^{\prime}\left(\bar{u}_{0}\right)=0 .
$$

The application of a preload $F_{0}$ results in a change of the total potential energy landscape due to the work performed by $F_{0}$. In particular, the two energy wells are no longer symmetric but exhibit different energy levels (depending upon the direction and magnitude of $F_{0}$ ). Figure 12 shows the total potential energy

$$
E(u)=\psi\left(u+u_{0}\right)-\psi^{\prime}\left(u_{0}\right) u
$$

for positive and negative predeformation $u_{0}$. 


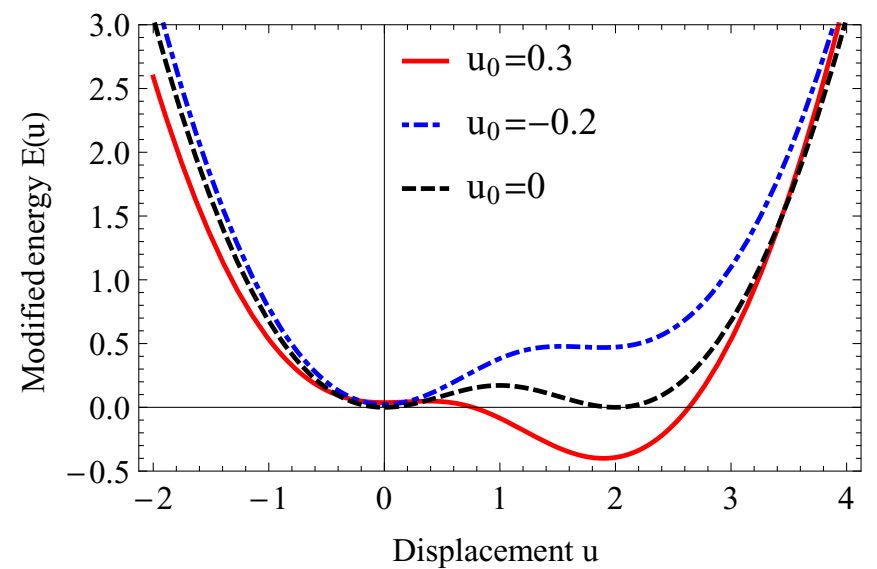

FIG. 12. (Color online) Energy landscape $E(u)$ with positive and negative predeformation as well as without preloads.

When the force $F_{0}$ points away from the direction of snapping, it leads to an unstable kink that disintegrates into ripples about the initial equilibrium point and does not show interesting wave propagation phenomena (yet it allows for the absorption of impact energy). However, when the preload is in the direction of snapping, the energy landscape becomes negative near its second well. To date, all models of the Frenkel-Kontorova type have dealt with potentials that are either fully positive or negative in the transition region. In contrast, the mechanical system studied here produces a potential that is partially positive and partially negative in the transition region. Even for a small amount of precompression (cf. Fig. 13) the stability of the kink increases and the effect of discreteness (i.e., phonon radiation) to lower the wave speed is reduced [97,98]. The $x$ - $t$ contour plot for the wave propagation shown in Fig. 13 confirms that the kink characteristic is now linear and hence the kink has stabilized as compared to the case without precompression; $\mathrm{cf}$. Fig. 10.

Increasing the precompression creates a combination of a kink and trailing oscillations that are localized as the wave moves through the chain. After attaining a certain width

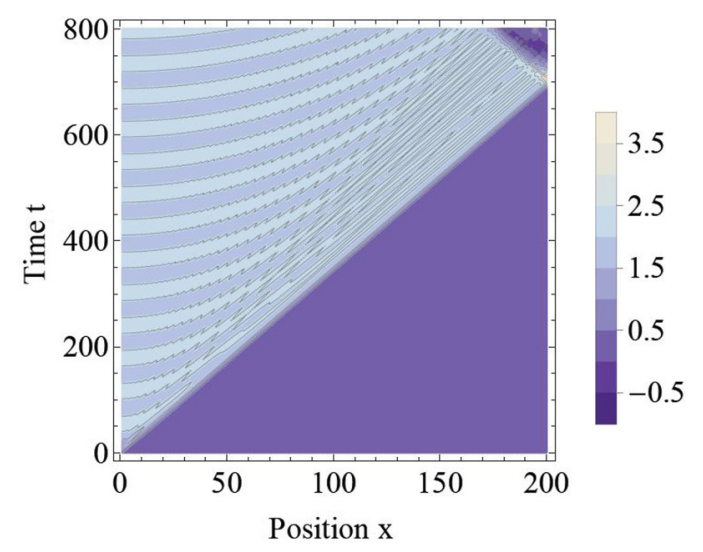

FIG. 13. (Color online) The $x$ - $t$ contour diagram of the numerical solution for a precompression $\bar{u}_{0}=0.03$. The kink characteristic is straighter than the characteristic without precompression.

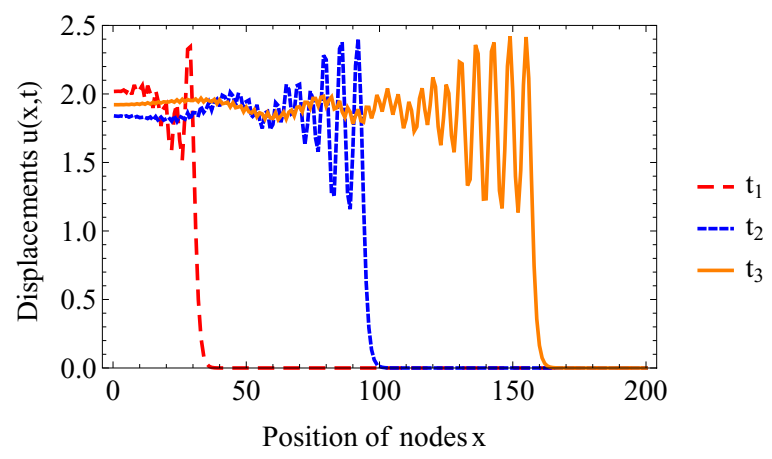

(a)

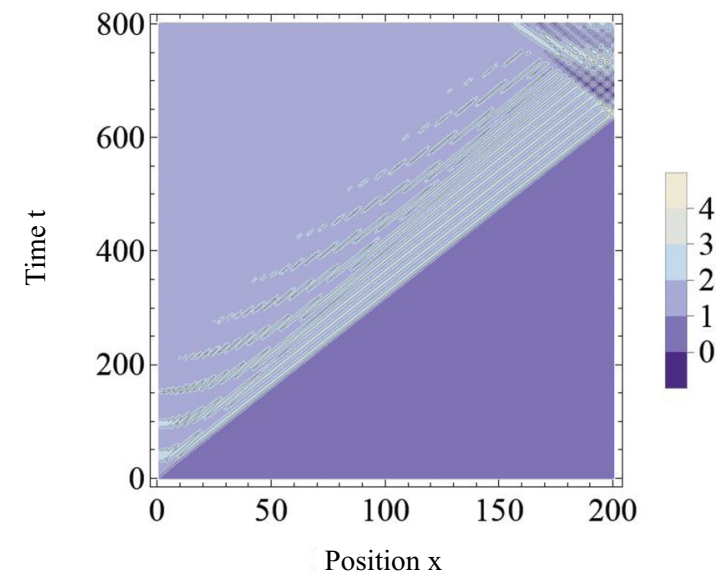

(b)

FIG. 14. (Color online) (a) Wave profiles for $\bar{u}_{0}=0.3$ and $t_{3}>t_{2}>t_{1}$. (b) The $x$ - $t$ contour diagram of the numerical solution for a precompression of $\bar{u}_{0}=0.3$.

of localization, the oscillations acquire a steady shape and propagate with the kink velocity. Figure 14 illustrates a typical wave profile and an $x-t$ contour diagram for the case of precompression (both have been obtained numerically). The oscillations forming in the wake of the kink are bounded by an envelope as in the case of the envelope soliton forming under medium amplitudes; see Sec. III B. This can be explained by the reduction in potential energy as each spring transitions from one energy well to the other. As shown in Fig. 12, a preload into the direction of snapping results in the snapped potential well having lower energy. Consequently, after snapping every mass has residual kinetic energy. This energy, however, is insufficient for a spring to snap back since part of the energy is carried away by the kink soliton. Hence, the masses oscillate in the snapped well with a medium amplitude giving rise to localization by modulational instability as seen in the envelope soliton case in Sec. III B. For these reasons, the predeformation results in a combination of the two modes of kink and envelope soliton propagation with an envelope solitonlike wave created behind the kink. As may be expected, the velocity of the propagating wave front increases with increasing precompression for a constant input of energy as shown in Fig. 15. There is a sharp rise in the velocity of propagation for small precompressions followed by a fairly linear increase for large precompressions, ultimately becoming supersonic. 


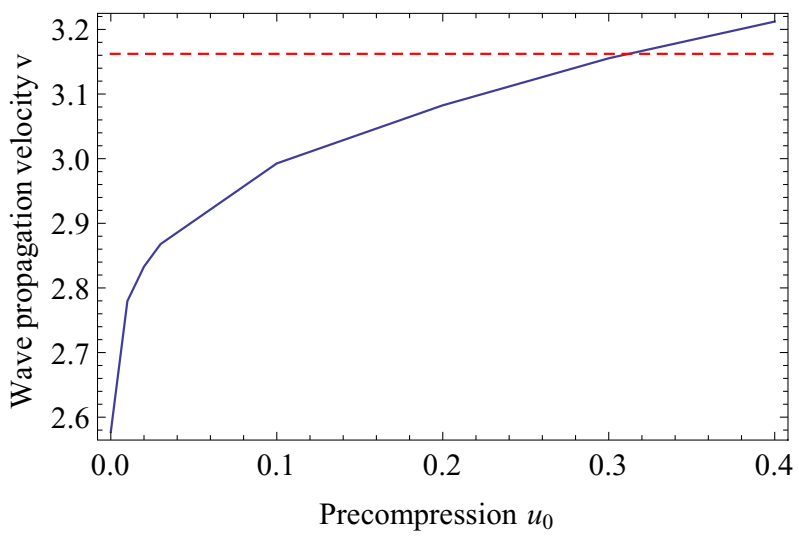

FIG. 15. (Color online) Variation of the kink propagation velocity with precompression for an initial (normalized) velocity of the first node of $v_{0}=4$. The dotted line shows the characteristic sound speed $\bar{c}_{0}$ of the medium for comparison.

\section{CONCLUSION}

We have shown that mechanical chains of bistable nonlinear-elastic elements offer a rich dynamic response with distinct regimes of wave propagation depending on the excitation amplitude. For small amplitudes, the chain propagates elastic waves in the linear regime characterized by wave dispersion. For moderate amplitudes, owing to modulational instability, an envelope soliton forms and propagates. For large amplitudes, the strongly nonlinear chain shows topological kink solitary waves. In all three regimes, we have derived analytical solutions of the displacement field in the continuum limit and we have demonstrated excellent agreement with the numerical solution obtained from a discrete chain of bistable elements. Wave propagation characteristics can be controlled by fine-tuning the geometric details of the bistable elements. Moreover, precompression brings the bistable elements closer to their snapping instability and hence can be used to control the wave propagation. We discussed the influence of precompression in all three amplitude regimes.

Our results highlight opportunities to design periodic mechanical structures and metamaterials containing bistable (negative-stiffness) elements that give rise to beneficial dynamic performance. This closes a gap between previous research on composite systems with (static) negative-stiffness phases and the nonlinear dynamics of structures undergoing large elastic deformation. Here the negative-stiffness effect (i.e., the unstable branch of the force-displacement curve) is utilized to create and propagate solitary-wave packages, which presents a way to achieve controllable metamaterial performance through negative-stiffness elements, while previous research in negative-stiffness materials mainly focused on their elastic and viscoelastic effective properties well within the realm of linearized kinematics. We deliberately chose a simple (possibly the simplest) elastic system to show the sought effects while allowing for closed-form analytical solutions, a rare find in nonlinear dynamics. The same qualitative response can be expected from various structural systems that allow for practical implementation. For example, the bistable spring elements can be replaced by buckled columns or membranes and the elastic springs connecting the bistable elements by compliant fillers. Current research explores possibilities to demonstrate the reported findings experimentally. Further, extensions to two and three dimensions by using bistable or multistable elements (having two or more stable equilibrium configurations, respectively) are left for future work.

\section{ACKNOWLEDGMENTS}

The authors gratefully acknowledge financial support from the National Science Foundation (NSF) under Grant No. CMMI-1200319. D.M.K. acknowledges support from the NSF through CAREER Award No. CMMI-1254424.
[1] B. Banerjee, An Introduction to Metamaterials and Waves in Composites (CRC, Boca Raton, 2011).

[2] M. Oudich, M. Badreddine Assouar, and Z. Hou, Appl. Phys. Lett. 97, 193503 (2010).

[3] L.-Y. Wu, T.-Y. Chiang, C.-N. Tsai, M.-L. Wu, and L.-W. Chen, Appl. Phys. A 109, 523 (2012).

[4] A. Spadoni and C. Daraio, Proc. Natl. Acad. Sci. USA 107, 7230 (2010).

[5] D. Bigoni, S. Guenneau, A. B. Movchan, and M. Brun, Phys. Rev. B 87, 174303 (2013).

[6] Y. Zhang, J. Wen, H. Zhao, D. Yu, L. Cai, and X. Wen, J. Appl. Phys. 114, 063515 (2013).

[7] B.-I. Popa, L. Zigoneanu, and S. A. Cummer, Phys. Rev. B 88, 024303 (2013).

[8] D. Miller, Opt. Express 14, 12457 (2006).

[9] G. Milton, M. Briane, and J. Willis, New J. Phys. 8, 248 (2006).

[10] M. Sigalas and E. Economou, Solid State Commun. 86, 141 (1993).
[11] M. S. Kushwaha, P. Halevi, L. Dobrzynski, and B. DjafariRouhani, Phys. Rev. Lett. 71, 2022 (1993).

[12] M. Ruzzene and F. Scarpa, Phys. Status Solidi B 242, 665 (2005).

[13] Z. Liu, X. Zhang, Y. Mao, Y. Zhu, Z. Yang, C. Chan, and P. Sheng, Science 289, 1734 (2000).

[14] P. Sheng, X. Zhang, Z. Liu, and C. Chan, Physica B 338, 201 (2003).

[15] D. Yu, Y. Liu, H. Zhao, G. Wang, and J. Qiu, Phys. Rev. B 73, 064301 (2006)

[16] J. Sánchez-Dehesa, D. Torrent, and L.-W. Cai, New J. Phys. 11, 013039 (2009).

[17] N. Fang, D. Xi, J. Xu, M. Ambati, W. Srituravanich, C. Sun, and X. Zhang, Nat. Mater. 5, 452 (2006).

[18] Z. Liu, C. T. Chan, and P. Sheng, Phys. Rev. B 71, 014103 (2005).

[19] Z. Yang, J. Mei, M. Yang, N. H. Chan, and P. Sheng, Phys. Rev. Lett. 101, 204301 (2008). 
[20] Y. Ding, Z. Liu, C. Qiu, and J. Shi, Phys. Rev. Lett. 99, 093904 (2007).

[21] B. Budiansky, Advances of Applied Mechanics (Elsevier, Amsterdam, 1974), pp. 1-65.

[22] T. Jaglinski and R. Lakes, in Adaptive Structures, edited by D. Wagg, I. Bond, P. Weaver, and M. Friswell (Wiley, New York, 2007), pp. 231-246.

[23] T. Jaglinski, D. Kochmann, D. Stone, and R. S. Lakes, Science 315, 620 (2007).

[24] E. Fatuzzo and W. Merz, Ferroelectricity (North-Holland, Amsterdam, 1967).

[25] A. Dorfmann and R. Ogden, Acta Mech. 174, 167 (2005).

[26] H. Sehitoglu, I. Karaman, R. Anderson, X. Zhang, K. Gall, H. J. Maier, and Y. Chumlyakov, Acta Mater. 48, 3311 (2000).

[27] P. M. Chaplya and G. P. Carman, Proc. SPIE 4699, 124 (2002).

[28] D. Platus, Vib. Control Microelectron. Opt. Metrol. 1619, 44 (1992).

[29] R. S. Lakes, T. Lee, A. Bersie, and Y. C. Wang, Nature (London) 410, 565 (2001).

[30] G. Kirchhoff, J. Reine Angew. Math. 56, 285 (1859).

[31] D. Kochmann and W. Drugan, J. Mech. Phys. Solids 57, 1122 (2009).

[32] D. M. Kochmann, Phys. Status Solidi B 249, 1399 (2012).

[33] T. Jaglinski, P. Frascone, B. Moore, D. S. Stone, and R. S. Lakes, Philos. Mag. 86, 4285 (2006).

[34] Y. Wang and R. Lakes, J. Appl. Phys. 90, 6458 (2001).

[35] C. S. Wojnar and D. M. Kochmann, Phys. Status Solidi B 251, 397 (2014).

[36] W. J. Drugan, Phys. Rev. Lett. 98, 055502 (2007).

[37] D. Kochmann and W. Drugan, Proc. R. Soc. London Ser. A 468 , 2230 (2012).

[38] C. S. Wojnar and D. M. Kochmann, Philos. Mag. 94, 532 (2014).

[39] C.-M. Lee, V. Goverdovskiy, and A. Temnikov, J. Sound Vib. 302, 865 (2007).

[40] C.-M. Lee and V. Goverdovskiy, J. Sound Vib. 331, 914 (2012).

[41] L. Kashdan, C. Seepersad, M. Haberman, and P. Wilson, Rapid Prototyping J. 18, 194 (2012).

[42] Y. Frenkel and T. Kontorova, Phys. Z. Sowjetunion 13, 1 (1938).

[43] O. M. Braun and Y. S. Kivshar, Phys. Rep. 306, 1 (1998).

[44] M. Remoissenet and M. Peyrard, Phys. Rev. B 29, 3153 (1984).

[45] M. Peyrard and M. Remoissenet, Phys. Rev. B 26, 2886 (1982).

[46] W. Atkinson and N. Cabrera, Phys. Rev. 138, A763 (1965).

[47] N. Flytzanis, S. Crowley, and V. Celli, J. Phys. Chem. Solids 38, 539 (1977).

[48] F. C. Frank and J. H. van der Merwe, Proc. R. Soc. London Ser. A 198, 205 (1949).

[49] F. C. Frank and J. H. van der Merwe, Proc. R. Soc. London Ser. A 198, 216 (1949).

[50] F. C. Frank and J. H. van der Merwe, Proc. R. Soc. London Ser. A 200, 125 (1949).

[51] O. M. Braun and V. K. Medvedev, Sov. Phys. Usp. 32, 328 (1989).

[52] F. Liu and M. G. Lagally, Phys. Rev. Lett. 76, 3156 (1996).

[53] S. C. Erwin, A. A. Baski, L. J. Whitman, and R. E. Rudd, Phys. Rev. Lett. 83, 1818 (1999).

[54] S. V. Dmitriev, T. Shigenari, A. A. Vasiliev, and K. Abe, Phys. Rev. B 55, 8155 (1997).
[55] S. V. Dmitriev, T. Shigenari, and K. Abe, Phys. Rev. B 58, 2513 (1998).

[56] J. F. Currie, A. Blumen, M. A. Collins, and J. Ross, Phys. Rev. B 19, 3645 (1979).

[57] J. Pouget and G. A. Maugin, Phys. Rev. B 31, 4633 (1985).

[58] J. Pouget and G. A. Maugin, Phys. Rev. B 30, 5306 (1984).

[59] P. Maffre and M. Peyrard, Phys. Rev. B 45, 9551 (1992).

[60] P. Maffre and M. Peyrard, J. Phys.: Condens. Matter 6, 4869 (1994).

[61] F. Fraternali, T. Blesgen, A. Amendola, and C. Daraio, J. Mech. Phys. Solids 59, 89 (2011).

[62] T. Blesgen, F. Fraternali, J. Raney, A. Amendola, and C. Daraio, Mech. Res. Commun. 45, 58 (2012).

[63] R. Lakes, P. Rosakis, and A. Ruina, J. Mater. Sci. 28, 4667 (1993).

[64] A. Cherkaev, E. Cherkae, and L. Slepyan, J. Mech. Phys. Solids 53, 383 (2005).

[65] L. Slepyan, A. Cherkaev, and E. Cherkae, J. Mech. Phys. Solids 53, 407 (2005).

[66] L. Slepyana and M. V. Ayzenberg-Stepanenkob, J. Mech. Phys. Solids 52, 1447 (2004).

[67] A. M. Balk, A. V. Cherkaev, and L. I. Slepyan, J. Mech. Phys. Solids 49, 131 (2001).

[68] A. M. Balk, A. V. Cherkaev, and L. I. Slepyan, J. Mech. Phys. Solids 49, 149 (2001).

[69] A. Vainchtein, J. Mech. Phys. Solids 58, 227 (2010).

[70] A. Vainchtein, Physica D 239, 1170 (2010).

[71] H. Schwetlick and J. Zimmer, SIAM J. Math. Anal. 41, 1231 (2009).

[72] M. Remoissenet, Phys. Rev. B 33, 2386 (1986).

[73] S. Pnevmatikos, N. Flytzanis, and M. Remoissenet, Phys. Rev. B 33, 2308 (1986).

[74] V. Nesterenko, Dynamics of Heterogeneous Materials (Springer, Berlin, 2001).

[75] B. F. Feeny and A. R. Diaz, J. Vib. Acoust. 132, 061013 (2010)

[76] C. Daraio, V. F. Nesterenko, E. B. Herbold, and S. Jin, Phys. Rev. Lett. 96, 058002 (2006).

[77] K. Hormann and J. Zimmer, J. Mech. Phys. Solids 55, 1385 (2007).

[78] I. Müller, Arch. Ration. Mech. Anal. 70, 61 (1979).

[79] I. Müller and P. Villaggio, Arch. Ration. Mech. Anal. 65, 25 (1977).

[80] F. Fraternali, L. Senatore, and C. Daraio, J. Mech. Phys. Solids 60, 1137 (2012).

[81] J. Yang, C. Silvestro, S. N. Sangiorgio, S. L. Borkowski, E. Ebramzadeh, L. D. Nardo, and C. Daraio, Smart Mater. Struct. 21, 012002 (2012).

[82] W. Parnell, IMA J. Appl. Math. 72, 223 (2007).

[83] K. Bertoldi and M. C. Boyce, Phys. Rev. B 78, 184107 (2008).

[84] A. C. Scott, Am. J. Phys. 37, 52 (1969).

[85] S. Dusuel, P. Michaux, and M. Remoissenet, Phys. Rev. E 57, 2320 (1998).

[86] M. Salerno, Physica D 17, 227 (1985).

[87] M. Cirillo, R. D. Parmentier, and B. Savo, Physica D 3, 565 (1981).

[88] K. Nakajima, T. Yamashita, and Y. Onodera, J. Appl. Phys. 45, 3141 (1974). 
[89] T. Dauxois and M. Peyrard, Physics of Solitons (Cambridge University Press, Cambridge, 1967), pp. 42-59.

[90] O. Klein, Z. Phys. 37, 895 (1926).

[91] A. D. Polyanin and V. F. Zaitsev, Handbook of Nonlinear Partial Differential Equations (Chapman and Hall/CRC, Boca Raton, 2004).

[92] A. H. Nayfeh, Introduction to Perturbation Techniques (Wiley, New York, 1993), pp. 166-168.
[93] V. E. Zakharov, J. Appl. Mech. Tech. Phys. 9, 190 (1968).

[94] V. E. Zakharov and A. Shabat, Zh. Eksp. Teor. Fiz. 61, 118 (1971) [Sov. Phys.-JETP 34, 62 (1972)].

[95] T. B. Benjamin and J. E. Feir, J. Fluid Mech. 27, 417 (1967).

[96] M. Remoissenet, Waves Called Solitons: Concepts and Experiments (Springer, Berlin, 1999).

[97] M. Peyrard and M. D. Kruskal, Physica D 14, 88 (1984).

[98] C. Kunz and J. A. Combs, Phys. Rev. B 31, 527 (1985). 